Int. J. Dev. Biol. 56: 693-699 (2012)

doi: $10.1387 / \mathrm{ijdb} .120049 \mathrm{~ms}$

\title{
Effect of RNAi-mediated knockdown of the Bombyx mori transformer-2 gene on the sex-specific splicing of Bmdsx pre-mRNA
}

\author{
MASATAKA G. SUZUKI*,1, KEIJI SUZUKI' ${ }^{1}$ FUGAKU AOKI ${ }^{1}$ and MASAHIRO AJIMURA ${ }^{2}$ \\ ${ }^{1}$ Department of Integrated Biosciences, Graduate School of Frontier Sciences, The University of Tokyo, Kashiwa and \\ ${ }^{2}$ National Institute of Agrobiological Sciences, Tsukuba, Ibaraki, Japan
}

\begin{abstract}
In Drosophila melanogaster, transformer-2 (tra-2) is essential for female differentiation and is known to induce female-specific splicing of doublesex (dsx). The function of Bmtra-2, the Bombyx mori homolog of tra-2, on the other hand remains to be elucidated. As an initial step to learn about the biological function of Bmtra-2, we determined whether Bmtra-2 is capable of inducing the female-specific splicing of Drosophila dsx. RNAi-mediated knockdown of Bmtra-2 using Bombyx cultured cells transiently transfected with a dsx minigene revealed that Bmtra-2 can induce female-specific splicing of Drosophila $d s x$. To examine the role Bmtra-2 plays in regulating sex-specific splicing of Bmdsx pre-mRNA, we used an RNAi approach to reduce BmTra-2 expression in the early embryo. Embryos injected with dsRNAs or siRNAs targeted to Bmtra-2 showed no variation in the sex-specific splicing pattern of Bmdsx pre-mRNA. RNAi knockdown of Bmtra-2 in the early embryo caused abnormal testis formation. Taken together, these results indicate that $B m t r a-2$ is required for normal testis development, but is not involved in regulating the sex-specific splicing of Bmdsx pre-mRNA, even though it is capable of inducing the female-specific splicing of Drosophila dsx.
\end{abstract}

KEY WORDS: sex determination, Bombyx mori, transformer-2, alternative splicing, doublesex

\section{Introduction}

In Drosophila melanogaster, tra-2 is a key gene that acts on the cascades regulating both somatic sexual differentiation and male fertility (Belote and Baker, 1983). In female somatic tissues, the tra-2 protein acts in concert with the transformer (tra) protein to bind to the cis-regulatory element (dsxRE) upstream of the $d s x$ gene in the female-specific exon. This activates the weak 3'splicing site preceding the exon and generates the female-type Dsx protein $\left(D s x^{F}\right)$, which regulates the downstream genes that allow female development to proceed. Although the tra-2protein is expressed in males, the absence of male Tra activity means that $d s x$ pre-mRNA is spliced by default to generate the male-type Dsx protein (Dsx ${ }^{\mathrm{M}}$ ), which in turn regulates genes necessary for male development (Ryner and Baker 1991). Moreover, tra-2, and tra also regulate the sex-specific splicing of fruitless (fru), the gene that controls sexual behavior and sex-specific nervous system differentiation
(Taylor and Knittel 1995). A similar function of tra-2 has been reported in several dipteran insects. RNAi-mediated knockdown of tra-2 in Musca (Burghardt et al., 2005), Ceratitis (Salvemini et al., 2009), and Anastrepha (Samo et al., 2010) caused disruption of endogenous tra-2 function in these species and the subsequent male-specific splicing of the endogenous $d s x$ pre-mRNAs, leading to the transformation of chromosomally female embryos into adult pseudomales. These findings raise the possibility that tra-2 function is conserved among dipteran insects.

tra-2 is also necessary for male germline function. Mutant males carrying loss-of-function tra2mutations are morphologically normal but display incomplete spermatogenesis and produce immotile sperm. As a result, these mutant males are sterile (Hazelrigg and Tu, 1994; Madigan et al., 1996; Mattox et al., 1996). The

Abbreviations used in this paper: Bmtra-2, Bombyx mori homolog of tra-2; dsx, doublesex; fru, fruitless; RE, regulatory element; RNAi, inhibitory RNA; tra, transformer.

*Address correspondence to: Masataka G. Suzuki. Department of Integrated Biosciences, Graduate School of Frontier Sciences, The University ofTokyo, 5-1-5 Kashiwanoha, Kashiwa-shi, Chiba-ken 277-8562, Japan.

Tel: +81-4-7136-3694. Fax: +81-4-7136-3698. e-mail: gakyo@k.u-tokyo.ac.jp - web: http://park.itc.u-tokyo.ac.jp/seigyo

Supplementary Material (one figure) for this paper is available at: http://dx.doi.org/10.1387/ijdb.120049ms

Accepted: 4 July 2012. Final, author-corrected PDF published online: 12 November 2012. Edited by: Makoto Asashima

ISSN: Online 1696-3547, Print 0214-6282

(C) 2012 UBC Press

Printed in Spain 
testis-specific splicing of pre-mRNAs from two genes expressed during spermatogenesis, exuperantia (exu) and alternative testis transcripts (att), are dependent on Tra-2 (Hazelrigg and Tu, 1994; Madigan et al., 1996). Mutations that affect the alternatively spliced 3' UTR of exu mRNA lead to a significant reduction in exu RNA accumulation in male germ cells. Null mutations in exu cause male sterility and lead to the formation of sperimatids with defects in nuclear elongation (Hazelrigg and Tu, 1994).

In the silkworm, B. mori, the chromosomal sex determination mechanism is distinct from that of $D$. melanogaster, with female $(\mathrm{ZW})$ being the heterogametic sex and male (ZZ) the homogametic sex. It has been shown genetically that female sex in $B$. mori is determined by the presence of a dominant feminizing factor on the W chromosome (Hashimoto, 1933). Moreover, no sex-specific regulatory $S x /$ homolog has been isolated from $B$. mori (Niimi et al., 2006). Also a tra homolog has not been found in the Bombyx genome (Mita et al., 2009). Despite these differences, a B. mori $d s x$ homolog $(B m d s x)$ has been implicated in sex determination (Suzuki et al., 2005). Bmdsx produces alternatively spliced mRNA isoforms that encode sex specific transcription factors as observed in dsx (Suzuki et al., 2001). However, the underlying mechanism for sex-specific splicing is clearly different between $d s x$ and $B m d s x$. We have demonstrated that female splicing of Bmdsx pre-mRNA represents the default mode when tested in HeLa nuclear extracts, and also that the female exon is devoid of putative TRA/TRA-2 binding sites (Suzuki et al., 2001). A splicing inhibitor, BmPSI is involved in the regulation of male-specific splicing of Bmdsx (Suzuki et al., 2008). Since BmPSI does not exhibit any sequence relationship to known SR proteins, such as Tra and Tra-2, the regulatory mechanism of sex-specific alternative splicing of $B m d s x$ is distinct from that of $d s x$.

The tra-2homolog of Bombyx mori(Bmtra-2) has been identified, but its function remains to be elucidated (Niu et al., 2005). In the present study, we used Bmtra-2 RNAi-mediated knockdown to determine whether Bmtra-2 is required for regulating Bmdsxpre-mRNA sex-specific splicing. In addition, we provide evidence indicating a possible role for Bmtra-2 in embryonic testis morphogenesis.

\section{Results}

\section{Bmtra-2 can induce the female-specific splicing of Drosophila dsx}

As an initial step to learn about the biological function of Bmtra-2, we examined whether Bmtra-2 is capable of inducing the femalespecific splicing of Drosophila ds $x$. For this purpose, RNAi-mediated knockdown of Bmtra-2was performed using Bombyxcultured cells (BmN cells) transiently transfected with a dsx minigene (Fig. 1A). In agreement with previous reports, female-type dsx mRNA was produced when the $d s x$ minigene was co-transfected with both tra and tra-2 cDNA constructs (Fig.1B, lane 4). Similar results were observed when the $d s x$ minigene was co-transfected with only the tra cDNA construct (Fig. 1B, lane 2). In this condition, the cells were subjected to RNAi experiments with Bmtra-2 dsRNA. Although we were not able to completely block Bmtra-2expression, a significant reduction of female-type $d s x m R N A$ was observed with a concomitant reduction in Bmtra-2 expression (Fig. 1B, lanes 5 and 6). These results indicate that Bmtra-2 can function in place of Drosophila tra-2 to induce the female-specific splicing of $d s x$. Male-type $d s x$ mRNA, which arises as the default in splicing, was observed in all cases.

\section{Downregulation of Bmtra-2 by RNAi does not affect the sex- specific splicing of $\mathrm{Bmdsx}$}

To further test whether Bmtra-2 plays a role in regulating sex-specific splicing of $B m d s x$ pre-mRNA, we utilized an RNAi approach to reduce Bmtra-2 expression in Bombyx individuals. Microinjection of dsRNA into embryos has most often been used for B. mori, and in all cases successfully, although silencing levels vary (Terenius et al., 2011). Therefore, we decided to use embryos for RNAi experiments. Eggs during the early embryonic stage (6-8 $\mathrm{hr}$ after oviposition), a developmental period known to be sensitive to RNAi-mediated gene knockdown (Yamaguchi et al., 2011), were injected with Bmtra-2 dsRNA. In addition, the effects of three siRNAs (si-29, si-30, and si-31) designed to target Bmtra-2 were also examined. Total RNA was extracted from each egg four days after injection and subjected to RT-PCR analysis. As shown in Fig. 2A, real-time RT-PCR confirmed a significant reduction in Bmtra-2 transcript levels in embryos injected with Bmtra-2dsRNA. Similarly, siRNA injections caused a significant reduction in Bmtra-2 mRNA levels, but those effects were slightly lower than that observed
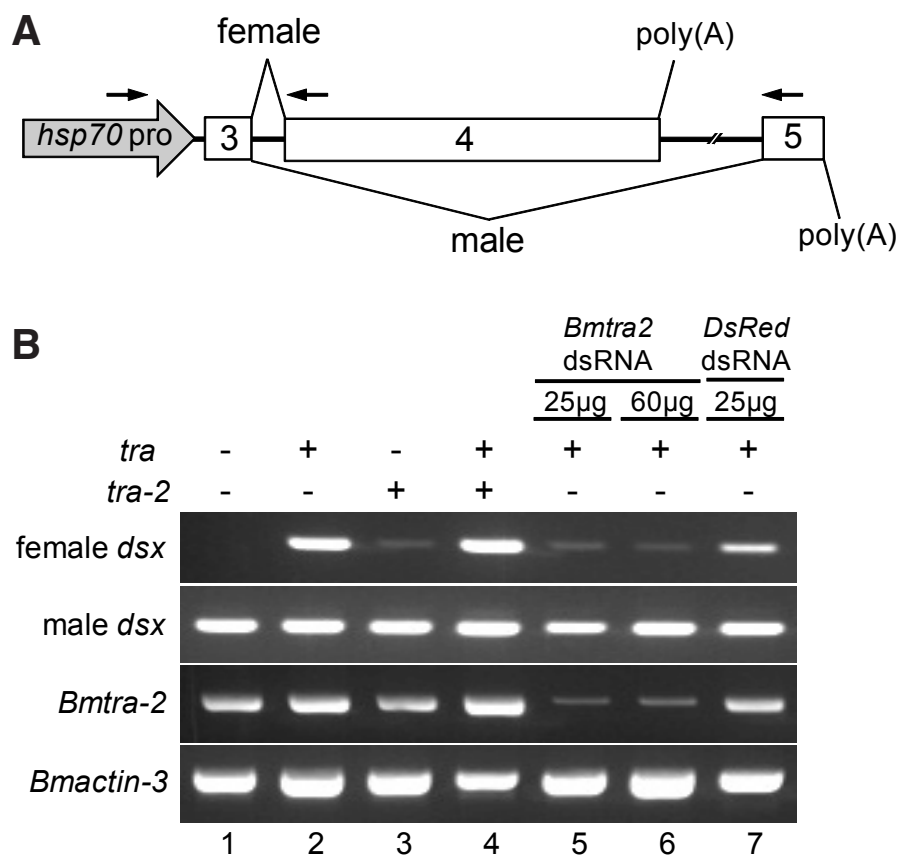

Fig. 1. Effect of RNAi-mediated Bmtra-2 knockdown on the sexspecific splicing of a dsx minigene using Bombyx cultured cells. (A) Structure of the dsx minigene construct used for assaying the sex-specific splicing of dsx in BmN cells. Boxes represent exons. The numbers above the diagram represent exon labels. V-shaped lines above (skipping of the female-specific exon) and below (inclusion of the female-specific exon) the diagram represent the endogenous dsx splice variants observed in males and females. Polyadenylation sites are indicated by Poly A. The arrows indicate the approximate location of the primers that were used for RT$P C R$ in (B). (B) The dsx minigene was co-transfected into BmN cells with $(+)$ or without (-) tra and tra-2 cDNA constructs. Female- or male-specific splicing was detected by RT-PCR and analyzed on a $2 \%$ agarose gel. Labels to the left of the gel refer to female splicing (dsx F) and male splicing (dsx M). Lower panel shows the down-regulation of Bmtra-2 expression in the dsRNA-treated cells. 
A
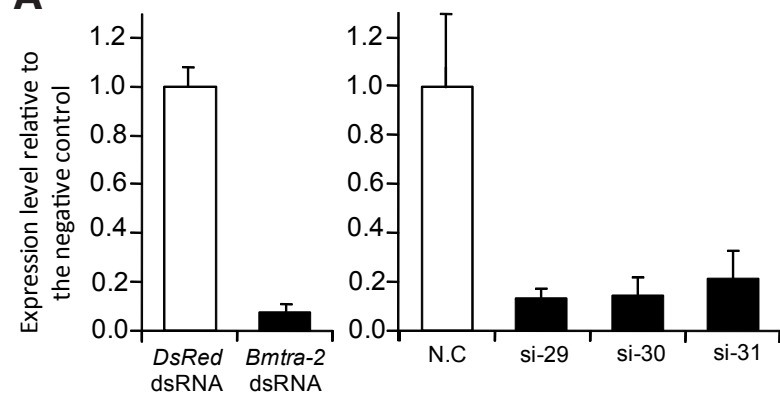

C

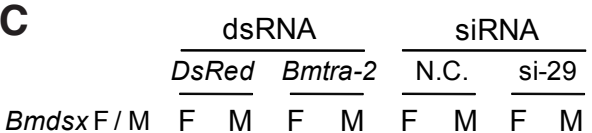

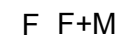
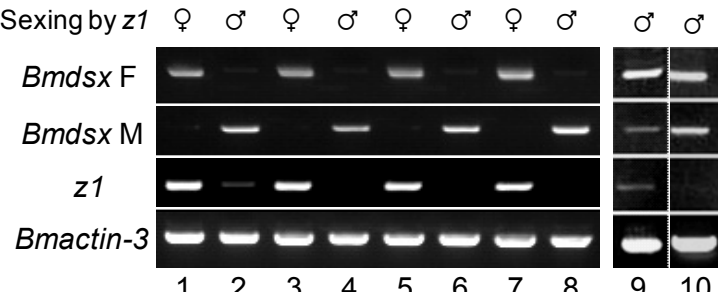

$\begin{array}{llllllll}1 & 2 & 3 & 4 & 5 & 6 & 7 & 8\end{array}$

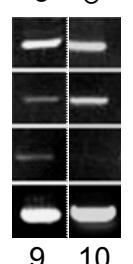

B
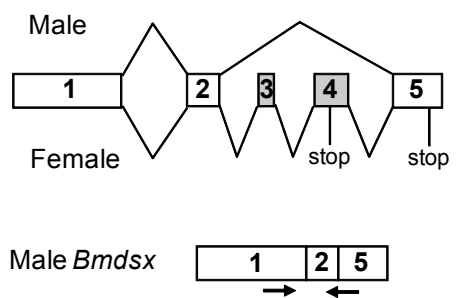

Female Bmdsx \begin{tabular}{c|c|c|c|c|}
\hline $\mathbf{1}$ & $\mathbf{2}$ & $\mathbf{3}$ & $\mathbf{4}$ & $\mathbf{5}$ \\
\hline
\end{tabular}

D

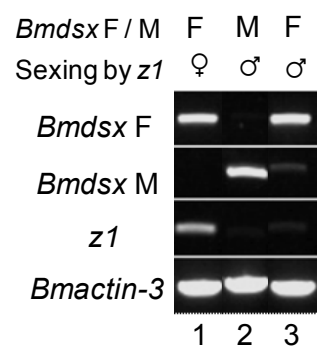

Fig. 2. Effect of RNAi-mediated Bmtra-2 knockdown on the sex-specific splicing of Bmdsx. (A) Quantification of Bmtra-2 mRNA level 4 days after injection using real-time $R T-P C R$. The elongation factor 2 (EF-2) was used as an internal control. Expression levels of Bmtra-2 mRNA in eggs injected with Bmtra-2 dsRNA or siRNAs were calculated relative to those of negative controleggs (shown as 1.00). Error bar, S.D.; $n=10$ individuals. (B) Schematic diagram of alternative splicing in Bmdsx pre-mRNA. Boxes represent exons. The gray region indicates the female-specific exons. The numbers in the diagram represent exon labels. V-shaped lines above (skipping of alternative exons) and below (inclusion of alternative exons) the diagram represent the endogenous Bmdsx splice variants observed in males and females. Stop codons are indicated by stop. The arrows indicate the approximate location of the primers that were used for RT-PCR in (C). (C) Female- or male-specific splicing was detected by RT-PCR and analyzed on a $2 \%$ agarose gel. Labels to the left of the gel refer to female splicing (Bmdsx F) and male splicing (Bmdsx M). Lower panel shows the results of molecular sexing by RT-PCR amplification of the transcript from the $\mathrm{z} 1$ gene, which is located on the $W$ chromosome. Bottom panel shows the results of RT-PCR amplification of the actin3 transcript, which served as a positive control for the RT-PCR reaction. (D) Abnormal expression of Bmdsx observed in untreated eggs.

with dsRNA. These results indicate that the injected dsRNA and siRNAs effectively silenced Bmtra-2 in the embryo. However, in most cases (90\%), downregulation of Bmtra-2 did not affect the sex-specific splicing of $B m d s x$, i.e. the expression pattern of $B m d s x$ was consistent with the sex of each embryo estimated by expression of the W chromosome specific gene $z 1$ (Ajimura et al., 2006) (Fig. 2C). Similar results were observed when the dsRNA mediated knockdown of Bmtra-2 were performed using Bombyx male and female cultured cells (see Supplementary Figure S1). Although five of the 51 examined embryos (10\%) showed abnormal expression of Bmdsx (i.e. the expression of female-type Bmdsx in male embryos) (Fig. 2C, lanes 9 and 10), this abnormality was also observed in untreated embryos (Fig. 2D, lane 3). This discrepancy may be explained by the accuracy of our molecular sexing. It was incomplete and sometimes the $z 1$ transcript was not amplified from embryos, which were identified as females by PCR using the female-specific SCAR marker. Its accuracy was approximately $97 \%$.
Taken together, these results suggest that even though Bmtra-2 can facilitate the female-specific splicing of $d s x$, it is not involved in the sex-specific splicing of Bmdsx.

\section{The Bmtra-2 B isoform is predominantly expressed in testis}

In Drosophila, tra-2pre-mRNA produces multiple mRNAs encoding three distinct isoforms of Tra-2 protein (Tra- $2^{264}$, Tra- $2^{226}$ and Tra-2 ${ }^{179}$ ) by alternative splicing (Mattox and Baker, 1991). Tra-2 $2^{226}$ and Tra-2 $2^{179}$ are specifically expressed in the male germline with Tra-2226 essential for both testis-specific exu splicing and male fertility (Mattox et al., 1996). Bmtra-2 pre-mRNA can also yield six alternatively spliced mRNAs encoding six distinct isoforms of BmTra-2 protein (Niu et al., 2005). To investigate which, if an, of these BmTra-2 isoforms are expressed in the male germline, RT-PCR analyses were performed with primer pairs reported by Niu et al., (2005) that amplify specific portions of each isoform. We found that most of the isoforms were ubiquitously expressed
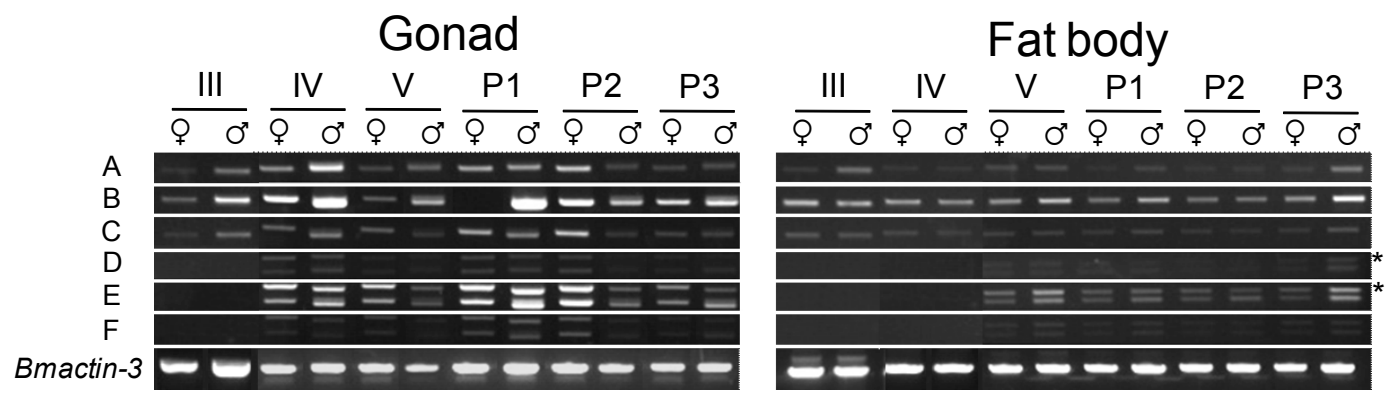

Fig. 3. RT-PCR analyses of the isoform-specific expression pattern of Bmtra-2. Total RNAs extracted from the gonad and fat body of males and females at different developmental stages were used as templates for RT-PCR reactions. Each of the isoforms (A-F) was amplified by RT-PCR using primer pairs reported by Niu et al., (2005) that can amplify specific portions of each isoform. III, IV and V indicate $3^{\text {rd }}, 4^{\text {th }}$ and $5^{\text {th }}$ instar larvae, respectively. P1, P3 and P5 indicate day-1, day-3 and day-5 pupae. RT-PCR amplification of the actin3 transcript was used as a positive control for RT-PCR reaction. Asterisks indicate unexpected transcripts that were not observed in the previous report described by Niu et al., (2005). 
in all of the tissues examined (Fig. 3). Isoform B mRNA, however, was predominantly expressed in the testis during the larval and early pupal stages with highest testis expression observed 1 day after pupation (P1). In addition, the expression levels of $A, C$, and $E$ isoforms were relatively higher in the ovary as compared with those in the testis at 3 days after pupation (P3). These results may imply that Bmtra-2 plays an important role in germline functions or gonadal development.

\section{Downregulation of Bmtra-2 in early embryo affects testis morphogenesis}

To evaluate whether Bmtra-2does indeed play an important role in germline functions or gonadal development, we injected Bmtra-2 dsRNA corresponding to a common region among the Bmtra-2 isoforms into pupae just after pupation. However, no reduction in Bmtra-2 transcripts was observed in pupae injected with the $\mathrm{Bm}$ tra-2 dsRNA even when the amount of injected dsRNA per pupa was increased to $50 \mu \mathrm{g}$ (data not shown). Therefore, we decided to use embryos for the RNAi experiments. Bmtra-2 dsRNA was injected into eggs laid by the sex-limited $p^{\text {sa }}$ (sable larval marking) strain, which enables us to discriminate females from males by a larval phenotypic marker (Tazima, 1943). Since this strain produces diapausing eggs, dsRNA was injected into eggs about 1 day after oviposition due to the necessity of using hydrochloric acid treatment to break diapause., Sexing of each larva was carried out at the $3^{\text {rd }}$ larval stage based on the sex-linked phenotypic marker, $p^{s a}$. The gonads were dissected out and were observed with a stereomicroscope. As shown in Fig. 4, Bmtra-2RNAi caused abnormal testis morphologies in three of the 21 larvae examined. The number of testicular lobes decreased in all cases (Fig. 4B,
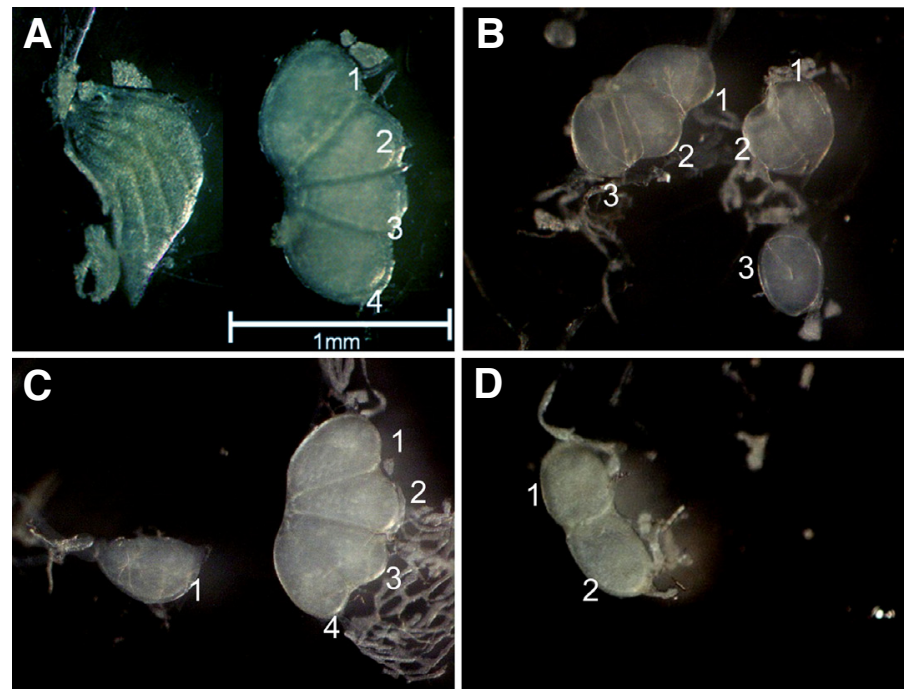

Fig. 4. Effect of RNAi-mediated Bmtra-2knockdown in the early embryo. dsRNAs were injected into eggs laid by the sex-limited $\mathrm{p}^{\mathrm{Sa}}$ (sable larval marking) strain approximately 1 day after oviposition., Gonads were dissected out during the $3^{\text {rd }}$ larval stage and observed with a stereomicroscope. (A) An ovary (left) and testis of a DsRed dsRNA injected individual. Note that a normal testis consists of four testicular lobes. Scale bar indicates $1 \mathrm{~mm}$. (B-D) Abnormal testes observed in the Bmtra-2 dsRNA injected individuals. The number of testicular lobes decreased in both testes (B). Although the right testis was normal, the left testis consisted of only one testicular lobe (C). The left testis consisted of only two testicular lobes while the right testis was non-existent (D).
$\mathrm{C}$, and D). Moreover, one of the testicular lobes was dissociated from the others (Fig. 4B). In the most extreme case, the testis on one side was completely absent (Fig. 4D). The remaining larvae were reared to adult moths and their fertilities investigated. All of the examined male and female moths had nearly wild-type levels of fertility (data not shown). Similar results were observed when siRNA (si29) was used in the microinjections (data not shown). These results indicate that Bmtra-2 has an important role in testis morphogenesis during the early embryonic stages.

\section{Discussion}

Here we have presented evidence that Bmtra-2 is not involved in the sex-specific splicing of Bmdsx despite being able to facilitate the female-specific splicing of $d s x$. Taken together with our previous research, which found that female splicing of Bmdsx premRNA represents the default mode when tested in HeLa nuclear extracts, and that the female exon is devoid of putative Tra/Tra-2 binding sites (Suzuki et al., 2001), it seems unlikely that Bmtra-2 participates in the regulation of sex determination. This situation is similar to tra-2 $\alpha$, a human homolog of tra-2. tra- $2 \alpha$ is not known to participate in the regulation of sex determination, but is capable of functionally replacing the endogenous Tra-2 protein when expressed in transgenic flies (Dauwalder et al., 1996). Previous studies have shown that the Tra- $2 \alpha$ protein is a sequence-specific activator of pre-mRNA splicing (Tacke et al., 1998). Tra-2 $\alpha$ binds to the exonic splicing enhancer on exon 4 of the gonadotropin-releasing hormone $(\mathrm{GnRH})$ gene and promotes the neuron-specific excision of intron A from the GnRH pre-mRNA together with $9 \mathrm{G} 8$ and SRp30 c (Seong et al., 2002). In this context, the protein level of Tra-2 $\alpha$ is strikingly high in adult mouse brain, but low or barely detected in other tissues (Chen et al., 2005). The scavenger receptor classB (SR-B) pre-mRNA can produce two alternatively spliced mRNAs encoding two distinct SR-B protein isoforms (SR-B type I and type II) in response to estrogen stimulation. Tra2 $\alpha$ and, in particular, Tra-2 $\beta$, another human homolog of tra-2, cause a shift from the SR-BI to SR-BII splice form (Zhang et al., 2007).

Tra- $2 \beta$ interacts with RBMY, which is a male germline RNA binding protein and potential alternative splicing regulator (Venables et al., 2000). This interaction modulates the splicing of several pre-mRNAs regulated by Tra-2 $\beta$ (Dreumont et al., 2010). For instance, two alternative exons in CREB and Acinus genes, which are efficiently included in the testis, are negatively regulated by Tra-2 $\beta$ and positively regulated by RBMY (Dreumont et al., 2010). Tra-2 $\beta$ also recapitulates testis-specific splicing of the homeodomain-interacting kinase HipK3 gene in a concentration-dependent manner and binds specifically to a long purine-rich sequence in the HipK3 testis-specific exon (Venables et al., 2005). In this context, theTra- $2 \beta$ protein is most highly expressed in mouse and human testes (Venables et al., 2000, 2005). These findings that Tra-2 $\beta$ is expressed particularly well in testis and that it modulates the testis-specific splicing of several genes are reminiscent of the functions of testis-specific Tra-2 isoforms that modulate testis-specific splicing of pre-mRNAs from two genes (exu and att) expressed during spermatogenesis. Therefore, an evolutionarily conserved role of Tra-2 protein seems likely to regulate testis-specific splicing of target pre-mRNAs.

Similar to tra-2and tra-2 $\beta$, one of the Bmtra-2 isoforms (Bisoform) was predominantly expressed in testis during the larval and early 
pupal stages (Fig. 3). Furthermore, downregulation of Bmtra-2 in early embryos caused abnormalities in testis morphology (Fig. 4B, $\mathrm{C}$ and D). These results indicate that Bmtra-2 has an important role in testis morphogenesis during the early embryonic stages. It is possible to hypothesize that the BmTra-2 B isoform may regulate testis-specific splicing of target genes that are crucial for testis morphogenesis. In the present study, the number of larvae showing abnormalities in testis was low (14.3\%). As described above, in this experiment, dsRNA was injected into eggs about 1 day after oviposition due to the necessity of using hydrochloric acid treatment to break diapause. Previous work has demonstrated that siRNA injection 1 or 2 days after oviposition causes a drastic decrease in the effectiveness of RNAi (Yamaguchi et al., 2011). Therefore, it seems possible that the low number of individuals with abnormal testis is due to the inappropriate timing of dsRNA injections.

Contrary to our findings that knockdown of Bmtra-2 caused abnormalities in testis morphology, complete loss of Tra-2 function in Drosophila does not affect testis development (Unni et al., 2003). Moreover, tra-2 is only required in Drosophila for sexual differentiation such as female determination, male courtship behavior, and spermatogenesis, but is dispensable for general viability (Unni et al., 2003). Differing from the Drosophila situation, functional orthologs of Tra-2 protein are thought to perform non-sex-specific functions essential for viability in both vertebrates (Seong et al., 2002) and nematodes (WormBase web site, http://www.wormbase. org, release WS98). These findings suggest that the biochemical activities and RNA recognition specificities of Tra-2 proteins in the fly and other organisms may be similar while the functions of the targeted mRNAs differ. It is therefore more suitable to consider the conserved function of Tra-2 as a tissue-specific alternative splicing factor rather than as a sex determination factor. The recruitment of Tra-2 into the sex determination pathway in Drosophila may reflect the rapid evolution of sex-determining mechanisms. Although the proteins in the sex determination pathways often show high sequence similarities, their participation in sexual differentiation is often not conserved (Marin and Baker, 1998). In fact, the DM domain proteins, DSX- and MAB-3-related transcription factors (DMRTs), are the only known examples of proteins that control sex determination or sexual differentiation in a broad array of metazoan animals (Matsuda et al., 2002).

As discussed above, an evolutionarily conserved role of the Tra-2 protein seems likely to regulate testis-specific splicing of genes expressed in testis or male germline. Consistent with this hypothesis, our findings in this study indicate that Bmtra-2 has an important role in testis morphogenesis during the early embryonic stages. At present, we cannot exclude the possibility that Bmtra-2 is also necessary for spermatogenesis since we were unable to successfully utilize RNAi-mediated knockdown to repress the expression of Bmtra-2 during the larval and pupal stages. However, because Bmtra-2 does not encode a protein isoform corresponding to the testis-specific Tra-2 isoforms, Tra- $2^{226}$ and Tra- $2^{179}$, which are essential for spermatogenesis (Niu et al., 2005), it can be assumed that Bmtra-2 is not involved in Bombyx spermatogenesis. In addition, Bmtra-2 may play an important role in female germline function or ovary development since the expression levels of $A, C$, and $E$ isoforms were relatively higher in the ovary as compared with those in the testis at 3 days after pupation (Fig. 3). A full understanding of the function of Bmtra-2 in the silkworm awaits further investigation.

\section{Materials and Methods}

\section{Plasmid construction}

The copia-dsx minigene, hsp-tra and hsp-tra-2 cDNA constructs were kindly provided by Prof. Kunio Inoue of Kobe University. The dsx genomic sequence was excised from the copia-dsx minigene construct and was cloned into the pHE-N vector multiple cloning site (Imai et al., 2005) to allow expression of the Drosophila dsx gene in Bombyx cultured cells. $1 \mu \mathrm{g}$ of the hsp-dsx minigene was transfected into $\mathrm{BmN}$ cells with or without $1 \mu \mathrm{g}$ of hsp-tra or $1 \mu \mathrm{g}$ of hsp-tra2 as shown in Fig. 1 according to the protocol described previously (Suzuki et al., 2008).

\section{Silkworm strains}

The Bombyx mori non-diapause and white egg strain, pnd-w1, was kindly provided by Dr. Ken-ichi Moto of RIKEN, and the sex-limited $p^{\text {sa }}$ (sable larval marking) strain, was provided by Dr. Takeshi Yokoyama from the Tokyo University of Agriculture and Technology. The developing eggs were incubated at $25^{\circ} \mathrm{C}$ with sufficient humidity completely enclosed in a plastic case. Larvae were reared on an artificial diet (Nihon Nosan) at approximately $25^{\circ} \mathrm{C}$.

\section{Preparation of dsRNAs and siRNAs}

Sequence conserved between the Bmtra-2 isoforms was amplified with primers TRA2dsRNAF2 (5'- CCG GAT CCT AAT ACG ACT CAC TAT AGG GCG ATT TGT GTC TTC AAC GTG C-3') and TRA2dsRNAR3 (5'- CCG GAT CCT AAT ACG ACT CAC TAT AGG GCG TAG GAG CGC TCC CGC TCG TAG -3'). Both primers contained a T7 promoter site (underlined). The amplified products were separated on a $2 \%$ agarose gel. A band of the appropriate size was excised and purified using a Wizard SV Gel and PCR Clean-Up System (Promega). The purified product was used as a template for in vitrotranscription. Complementary RNAstrands were transcribed using an AmpliScribe T7 Transcription Kit (Cellscript) and annealed by heating to $95^{\circ} \mathrm{C}$ for $1 \mathrm{~min}$ and then allowing to cool to room temperature. The quality of the dsRNA was examined by electrophoresis on a $2 \%$ agarose gel. The indicated amounts of dsRNAs were transfected into BmN cells according to the protocol described previously (Suzuki et al., 2008).

Three siRNAs targeted to Bmtra-2 (siRNA29, siRNA30, siRNA31) were designed using BLOCK-iT RNAi Designer (https://rnaidesigner.invitrogen. com/rnaiexpress/). The sequences of the siRNAs are as follows: siRNA29: sense- GCA CCG GAA UGG AAA UUG Att, antisense- UGA AUU UCC AUU CCG GUG Cat; siRNA30: sense- GCA GAU AAA CCA UAU CUU tt, antisense- AAA GAU AUG GUU UAU CUG Ctg; siRNA31: sense- CCG CGU CGA UUA UUC CAU Att, antisense- UAU GGAAUAAUC GAC GCG Gat. Each siRNAwas synthesized using the Custom Select siRNA synthesis service provided by Ambion. Silencer Negative Control \#1 siRNA (Ambion) was used as a negative control in the siRNA experiments.

\section{Transfection of DNA and dsRNAs into cells}

$1 \mu \mathrm{g}$ of the hsp-dsx minigene was transfected into BmN cells with or without $1 \mu \mathrm{g}$ of hsp-tra or $1 \mu \mathrm{g}$ of hsp-tra2 as shown in Fig. 1 according to the protocol described previously (Suzuki et al., 2008). To examine whether Bmtra-2 is capable of inducing the female-specific splicing of Drosophila $d s x$, the indicated amounts of Bmtra-2 dsRNA were transfected into BmN cells as described previously (Suzuki et al., 2008). Two days after dsRNA transfection, the cells were transfected with the hsp-dsx minigene $(1 \mu \mathrm{g})$ together with hsp-tra $(1 \mu \mathrm{g})$. Poly $(\mathrm{A})+\mathrm{RNA}$ was isolated $48 \mathrm{~h}$ after transfection using a Micro-FastTrack ${ }^{\mathrm{TM}} 2.0 \mathrm{mRNA}$-isolation kit (Invitrogen) according to the protocol provided by the manufacturer.

\section{Injection of dsRNAs and siRNAs into eggs}

dsRNAs and siRNAs were injected into eggs according as described by Yamaguchi et al., (2011) with a slight modification. Briefly, eggs were aligned in the same direction and glued onto the surface of glass slides covered with liquid glue (Arabic Yamato, YAMATO). The eggs were perforated on the ventral side with a $0.5 \mu \mathrm{m}$ diameter tungsten probe tip (Micro 
TABLE 1

PRIMER SEQUENCES AND PCR CONDITIONS UTILIZED IN THIS STUDY

\begin{tabular}{|c|c|c|c|c|c|c|}
\hline gene & primers & sequence & denaturation & annealing & elongation & $\mathrm{n}^{\circ}$ cycles \\
\hline \multirow{2}{*}{ Bmtra-2 } & BmTra2F1 & ATGTCTGATCGAGAGAGAAGTCGTTC & \multirow{2}{*}{$98^{\circ} \mathrm{C} 10 \mathrm{sec}$} & \multirow{2}{*}{$55^{\circ} \mathrm{C} 30 \mathrm{sec}$} & \multirow{2}{*}{$72^{\circ} \mathrm{C} 60 \mathrm{sec}$} & \multirow{2}{*}{26} \\
\hline & BmTra2R1 & AGCGCTCCCGCTCGTAGCGTC & & & & \\
\hline \multirow{2}{*}{$B m d s x \mathrm{~F}$} & FDSXF2 & CGCCTTACCGCAGACAGGCAG & \multirow{2}{*}{$98^{\circ} \mathrm{C} 10 \mathrm{sec}$} & \multirow{2}{*}{$57^{\circ} \mathrm{C} 30 \mathrm{sec}$} & \multirow{2}{*}{$72^{\circ} \mathrm{C} 60 \mathrm{sec}$} & \multirow{2}{*}{35} \\
\hline & E3E4R1 & AATTTCCAGCATTTTCTGGCG & & & & \\
\hline \multirow{2}{*}{ Bmdsx M } & FDSXF2 & CGCCTTACCGCAGACAGGCAG & \multirow{2}{*}{$98^{\circ} \mathrm{C} 10 \mathrm{sec}$} & \multirow{2}{*}{$57^{\circ} \mathrm{C} 30 \mathrm{sec}$} & \multirow{2}{*}{$72^{\circ} \mathrm{C} 60 \mathrm{sec}$} & \multirow{2}{*}{35} \\
\hline & E2E5R & TGCATCATCCAATAACCTTCG & & & & \\
\hline \multirow{2}{*}{ Bmactin-3 } & BA3F1 & AGATGACCCAGATCATGTTCG & \multirow{2}{*}{$98^{\circ} \mathrm{C} 10 \mathrm{sec}$} & \multirow{2}{*}{$55^{\circ} \mathrm{C} 30 \mathrm{sec}$} & \multirow{2}{*}{$72^{\circ} \mathrm{C} 40 \mathrm{sec}$} & \multirow{2}{*}{26} \\
\hline & BA3R1 & GAGATCCACATCTGTTGGAAG & & & & \\
\hline \multirow{2}{*}{ Female $d s x$} & hspF1 & CAACTACTGAAATCTGCCAAG & \multirow{2}{*}{$98^{\circ} \mathrm{C} 10 \mathrm{sec}$} & \multirow{2}{*}{$57^{\circ} \mathrm{C} 10 \mathrm{sec}$} & \multirow{2}{*}{$72^{\circ} \mathrm{C} 30 \mathrm{sec}$} & \multirow{2}{*}{30} \\
\hline & DmdsxFR2 & ATTCACAACGTATTGGCCCTC & & & & \\
\hline \multirow{2}{*}{ Male $d s x$} & $\mathrm{hspF} 1$ & CAACTACTGAAATCTGCCAAG & \multirow{2}{*}{$98^{\circ} \mathrm{C} 10 \mathrm{sec}$} & \multirow{2}{*}{$57^{\circ} \mathrm{C} 10 \mathrm{sec}$} & \multirow{2}{*}{$72^{\circ} \mathrm{C} 30 \mathrm{sec}$} & \multirow{2}{*}{30} \\
\hline & DmdsxMR2 & ATTTATTTCCACTCGAGCCTC & & & & \\
\hline \multirow{2}{*}{ Bmtra-2 A } & Bmtra2L & GCAGAAAATATAGAATCCCCATG & \multirow{12}{*}{$94^{\circ} \mathrm{C} 15 \mathrm{sec}$} & & & \\
\hline & Bmtra2R11 & CTAAGTTTACATTATAGAGGCGTG & & & & \\
\hline \multirow{2}{*}{ Bmtra-2 B } & Bmtra2M & GCAGAAAATATAGAAGCCCGACA & & & & \\
\hline & Bmtra2R11 & CTAAGTTTACATTATAGAGGCGTG & & & & \\
\hline & Bmtra2S & GCAGAAAATATAGAATCCCATC & & & & \\
\hline Bmtra-2C & Bmtra2R11 & CTAAGTTTACATTATAGAGGCGTG & & & & \\
\hline Remtra & Bmtra2L & GCAGAAAATATAGAATCCCCATG & & $55^{\circ} \mathrm{C} 30 \mathrm{sec}$ & $12^{2} \mathrm{Cos} \mathrm{sec}$ & 26 \\
\hline Bmtra-<U & LBmtra2R22 & GTCGGAAGATAAAGAATATGATC & & & & \\
\hline Bmtra-2F & Bmtra2M & GCAGAAAATATAGAAGCCCGACA & & & & \\
\hline Bmtra-< $\mathrm{E}$ & LBmtra2R22 & GTCGGAAGATAAAGAATATGATC & & & & \\
\hline & Bmtra2S & GCAGAAAATATAGAATCCCATC & & & & \\
\hline Bmtra-2ト & LBmtra2R22 & GTCGGAAGATAAAGAATATGATC & & & & \\
\hline
\end{tabular}

Support Corp.). Injections were performed using a glass capillary (GN-1C, ST Science). Total RNA was extracted from each egg 4 days after injection using Isogen (Nippon Gene).

\section{RT-PCR analyses}

First-strand cDNAs were synthesized from $500 \mathrm{ng}$ of poly (A)+RNAs extracted from the RNAi-treated cells using a PrimeScript cDNA synthesis kit (TaKaRa Bio Inc.) according to the protocol provided by the manufacturer with random hexamers as primers. Total RNA $(1 \mu \mathrm{g})$ extracted from the RNAi-treated eggs was transcribed using the same cDNA synthesis kit with the oligo-dT primer included in the kit. An aliquot (1/20th of the resulting reaction) was used for PCR using EmeraldAmp PCR Master Mix (TaKaRa Bio Inc.). The primer sequences and PCR conditions utilized in this study are indicated in Table 1. To perform molecular sexing of each egg, the $\mathrm{W}$ chromosome specific gene $z 1$ was amplified using primers $z 1-3$ and z1-11 (Ajimura et al., 2006). PCR products were analyzed on a $2 \%$ agarose gel and visualized with ethidium bromide.

\section{Quantitative real-time $R T-P C R$}

Quantitative real-time RT-PCR assays were performed using SYBR Premix Ex Taq II (TaKaRa Bio Inc.) on a Thermal Cycler Dice Real Time System (TaKaRa Bio Inc.) according to the manufacture's instructions. QTBmtraF1 (5'- ATG TCT GAT CGA GAG AGA AGT CGT TC - 3') and QTBmtraR1 (5'- GCT GTG ACT ATG ACT GCG GCC GGA TG - 3') were used for the quantification of the Bmtra-2 gene expression. BmEF-2F1 (5'- TCG TAC CGT GAG ACC GTA GCT - 3') and BmEF-2R1 (5' - ATG TCC TCT GGC AGA CCA TCA - 3') were used for the quantification of elongation factor-2 (BmEF-2) as an internal standard. The threshold cycle (CT) value was normalized with the CT value of the BmEF-2 gene using Multiplate RQ software (TaKaRa Bio Inc.). The relative value of Bmtra-2 expression against $B m E F-2$ expression was obtained in triplicate and the ratio of Bmtra-2 expression calculated.

\section{Acknowledgements}

We are grateful to Dr. J. Joe Hull for his helpful comments on the manuscript. Prof. Kunio Inoue is gratefully acknowledged for providing the copia-dsx minigene, hsp-tra and hsp-tra-2 cDNA constructs. We also thank Dr. Ken-ich Moto and Dr. Takeshi Yokoyama for providing the silkworm strains. We are grateful to Haruka Ito for technical supports of microinjections. This work was supported by Grant-in-Aid for Scientific Research (B), 23380032, 2011.

\section{References}

AJIMURA, M., SAHARA, K., ABE, H., TAMURA, T., SHIMADA, T. and MITA, K. (2006). Are the zinc-finger motif genes, $z l$ and $z 20$, located in the $W$ chromosome involved in the sex-determination of the domesticated silkworm, Bombyx mori? Seventh International Workshop on the Molecular Biology and Genetics of the Lepidoptera. Publications Orthodox Academy of Crete, J Insect Sci 7: 4-5.

BELOTE, J. M., and BAKER, B. S. (1983). The dual functions of a sex determination gene in Drosophila melanogaster. Dev Biol 95: 512-517.

CHEN, X., LI, J., WU, K., HAN, Y. and XU, P. (2005). Tra2 $\alpha$ promotes RA induced neural differentiation of P19 cells. Neurochem Res 30: 271-275.

DAUWALDER, B., AMAYA-MANZANARES, F. and MATTOX, W. (1996). A human homologue of the Drosophila sex determination factor transformer-2has conserved splicing regulatory functions. Proc. Natl Acad Sci USA 93: 9004-9009.

DREUMONT, N., BOURGEOIS, C. F., LEJEUNE, F., LIU, Y., EHRMANN, I. E., ELLIOTT, D. J. and STÉVENIN, J. (2010). Human RBMY regulates germline-specific splicing events by modulating the function of the serine/arginine-rich proteins $9 \mathrm{G} 8$ and Tra2- $\beta$. J Cell Sci 123: 40-50.

HASHIMOTO, H. (1933). The role of the $W$ chromosome for sex determination in the silkworm, Bombyx mori. Jap J Genet 8: 245-258.

HAZELRIGG, T. and TU, C. (1994). Sex-specific processing of the Drosophila exuperantia transcript is regulated in male germ cells by the tra-2 gene. Proc Natl Acad Sci USA 91:10752-10756.

IMAI, N., MATSUMOTO, S. and KANG, W. (2005). Formation of Bombyx mori nucleopolyhedrovirus IE2 nuclear foci is regulated by the functional domains for oligomerization and ubiquitin ligase activity. J Gen Virol 86: 637-644.

MADIGAN, S. J., EDEEN, P., ESNAYRA, J. and MCKEOWN, M. (1996). Att, a target for regulation by Tra2 in the testes of Drosophila melanogaster, encodes alterna- 
tive RNAs and alternative proteins. Mol Cell Biol 16: 4222-4230.

MARIN, I. and BAKER, B. S. (1998). The evolutionary dynamics of sex determination. Science 281: 1990-1994.

MATSUDA, M., NAGAHAMA, Y., SHINOMIYA, A., SATO, T., MATSUDA, C., KOBAYASHI, T., MORREY, C. E., SHIBATA, N., ASAKAWA, S., SHIMIZU, N., HORI, H., HAMAGUCHI, S. and SAKAIZUMI, M. (2002). DMY is a Y-specific DM-domain gene required for male development in the medaka fish. Nature 417: $559-563$.

MATTOX, W. and BAKER, B. S. (1991). Autoregulation of the splicing of transcripts from the transformer-2 gene of Drosophila. Genes Dev 5: 786-796.

MATTOX, W., MCGUFFIN, M. E. and BAKER, B. S. (1996). A negative feedback mechanism revealed by functional analysis of the alternative isoforms of the Drosophila splicing regulator transformer-2. Genetics 143: 303-314.

MITA, K., KASAHARA, M., SASAKI, S., NAGAYASU, Y., YAMADA, T., KANAMORI, H., et al.,2009). The genome sequence of silkworm, Bombyx mori. DNARes 11:27-35.

NIIMI, T., SAHARA, K., OSHIMA, H., YASUKOCHI, Y., IKEO, K. and TRAUT, W. (2006). Molecular cloning and chromosomal localization of the Bombyx Sex-lethal gene. Genome 49: 263-268.

NIU, B. L., MENG, Z. Q., TAO, Y. Z., LU, S. L., WENG, H. B., HE, L. H., and SHEN, W. F. (2005). Cloning and alternative splicing analysis of Bombyx mori transformer-2 gene using silkworm EST database. Acta Biochim Biophys Sin 37: 728-736.

RYNER, L. C. and BAKER, B. S. (1991). Regulation of doublesexpre-mRNA processing occurs by 3'-splice site activation. Genes Dev 5: 2071-2085.

SEONG, J. Y., HAN, J., PARK, S., WUTTKE, W., JARRY, H. and KIM, K. (2002). Exonic splicing enhancer-dependent splicing of the gonadotropin-releasing hormone premessenger ribonucleic acid is mediated by Tra2 $\alpha$, a 40-kilodalton serine/arginine-rich protein. Mol Endocrinol 16: 2426-2438

SUZUKI, M. G., OHBAYASHI, F., MITA, K., and SHIMADA, T. (2001). The mechanism of sex-specific splicing at the doublesex gene is different between Drosophila melanogaster and Bombyx mori. Insect Biochem Mol Biol 31: 1201-1211.

SUZUKI, M. G., FUNAGUMA, S., KANDA, T., TAMURA, T., and SHIMADA, T. (2005). Role of the male BmDSX protein in the sexual differentiation of Bombyx mori. Evol Dev 7: 58-68.
SUZUKI, M. G., IMANISHI, S., DOHMAE, N., NISHIMURA, T., SHIMADA T. and MATSUMOTO, S. (2008). Establishment of a novel in vivo sex-specific splicing assay system to identify a trans-acting factor that negatively regulates splicing of Bombyx mori dsx female exons. Mol Cell Biol 28: 333-343.

TACKE, R., TOHHYAMA, M., OGAWA, S. and MANLEY, J. L. (1998). Human Tra2 proteins are sequence-specific activators of premRNA splicing. Cell93: 139-148.

TAYLOR, B. J., and L. M. KNITTEL. (1995). Sex-specific differentiation of a male specific abdominal muscle, the muscle of Lawrence, is abnormal in hydroxyureatreated and in fruitless male flies. Development 121: 3079-3088

TAZIMA, Y. (1943). Cytogenetical improvement of autosexing method with use of larval marking (I). J Seric Sci Jpn 14: 76-89. (In Japanese).

TERENIUS, O., PAPANICOLAOU, A., GARBUTt, J. S., ELEFTHERIANOS, I., HUVENNE, H., KANGINAKUDRU, S. et al., (2011). RNA interference in Lepidoptera: an overview of successful and unsuccessful studies and implications for experimental design. J Insect Physiol 57: 231-245.

UNNI, E., SU, S., ZRALY, C. B. and MATTOX, W. (2003). Analysis of a null mutation in the Drosophila splicing regulator Tra2 suggests its function is restricted to sexual differentiation. Genesis 37: 76-83.

VENABLES, J. P., ELLIOTT, D. J., MAKAROVA, O. V., MAKAROV, E. M., COOKE, H. J. and EPERON, I. C. (2000). RBMY, a probable human spermatogenesis factor, and other hnRNP G proteins interact with Tra2 $\beta$ and affect splicing. Hum Mol Genet 9, 685-694.

VENABLES, J. P., BOURGEOIS, C. F., DALGLIESH, C., KISTER, L., STEVENIN, J. and ELLIOTT, D. J. (2005). Up-regulation of the ubiquitous alternative splicing factor Tra2 $\beta$ causes inclusion of a germ cell-specific exon. Hum Mol Genet 14: 2289-2303.

YAMAGUCHI, J., MIZOGUCHI, T. and FUJIWARA, H. (2011). siRNAs induce efficient RNAi response in Bombyx mori embryos. PLoS One 6: e25469.

ZHANG, X., MOOR, A. N., MERKLER, K. A., LIU, Q. and MCLEAN, M. P. (2007). Regulation of alternative splicing of liver scavenger receptor class $B$ gene by estrogen and the involved regulatory splicing factors. Endocrinology 148: 5295-5304. 


\section{Further Related Reading, published previously in the Int. J. Dev. Biol.}

Ceratitis capitata transformer-2 gene is required to establish and maintain the autoregulation of Cctra, the master gene for female sex determination

Marco Salvemini, Mark Robertson, Benjamin Aronson, Peter Atkinson, Lino C. Polito and Giuseppe Saccone

Int. J. Dev. Biol. (2009) 53: 109-120

Masculinization of $\mathrm{XX}$ Drosophila transgenic flies expressing the Ceratitis capitata DoublesexM isoform

Giuseppe Saccone, Marco Salvemini, Attilio Pane and Lino C. Polito

Int. J. Dev. Biol. (2008) 52: 1051-1057

A scaleless wings mutant associated with tracheal system developmental deficiency in wing discs in the silkworm, Bombyx mori

Qingxiang Zhou, Shunming Tang, Yin Chen, Yongzhu Yi, Zhifang Zhang and Guifang Shen Int. J. Dev. Biol. (2004) 48: 1113-1117

The brain secretory peptides that control moulting and metamorphosis of the silkmoth, Bombyx mori.

$\mathrm{H}$ Ishizaki and A Suzuki

Int. J. Dev. Biol. (1994) 38: 301-310

Genes that are involved in Bombyx body plan and silk gene regulation.

Y Suzuki

Int. J. Dev. Biol. (1994) 38: 231-235

5 yr ISI Impact Factor $(2011)=2.959$

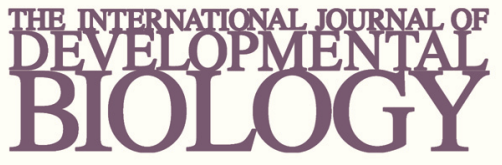

Volume 54 Nos. $6 / 7$
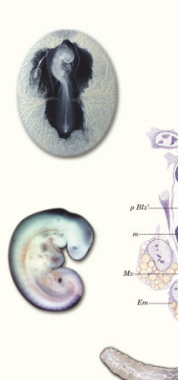

Developmental Hematopoiesis
Special Issue

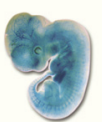

(2) (8)

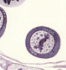

(2)
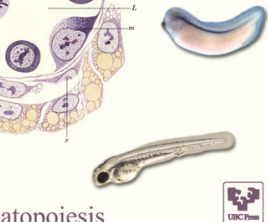

(5)

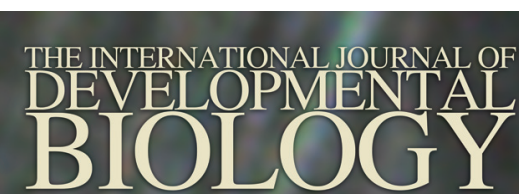

Volume 56 Nos. $1 / 2 / 3$

Special Issue

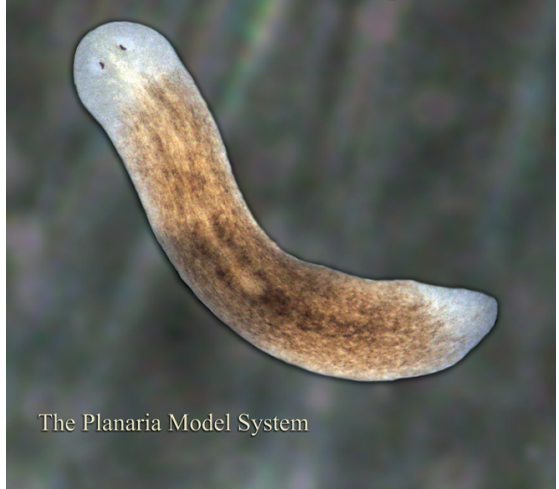

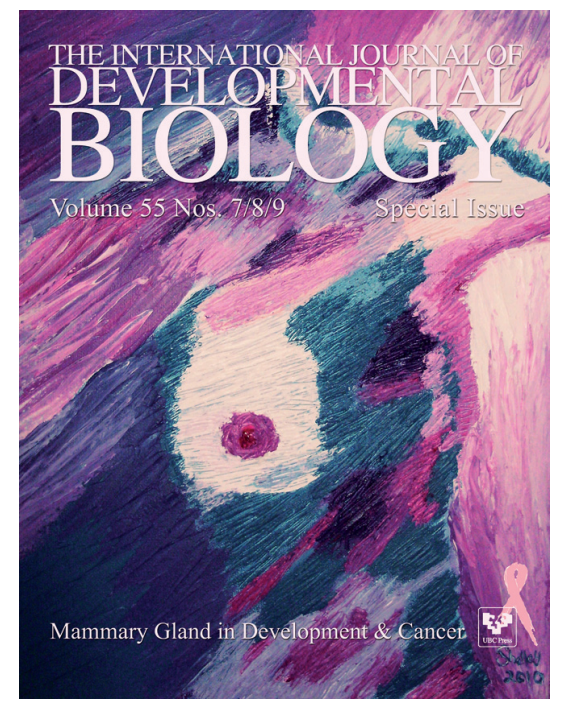

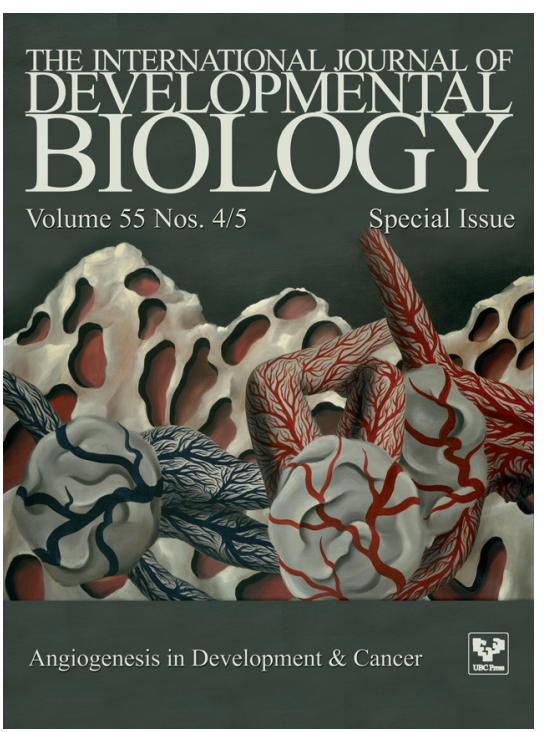

